\title{
HAS THE RELATIVITY PRINCIPLE IN THE SPECIAL THEORY OF RELATIVITY BEEN FULLY VERIFIED BY EXPERIMENTS?
}

\author{
WANG Ruyong, CHEN Zhongying, DONG Xianquan \\ Institute of Mechanics, Academia Sinica, Beijing, The People's Republic of China
}

Received 1 October 1979

Revised manuscript received 3 November 1979

\begin{abstract}
Up to now all experiments used to verify the special theory of relativity have been done with the earth as the reference system. A suggested new Michelson-Morley experiment in Space Lab will be the first to examine the relativity principle in an inertial system other than the earth.
\end{abstract}

According to the general point of view at present, it seems that the relativity principle in the special theory of relativity has been fully verified by experiments. But as a matter of fact, when analyzing the existing experiments used to verify special relativity, it can be seen that they all bear the following common feature: they were carried out at laboratories which were stationary with respect to the earth (such as Fizeau's experiment, Michelson-Morley's experiment, tests of the increase in mass with increase in speed, and the experiments which show the extension of the lifetime of a decaying particle moving at high speed), or they use as a comparison reference objects which are at rest relative to the earth (experiments with an atomic clock taken on an around-the-world airplane flight). That is to say, all verifying experiments were done with the earth as the reference system. Although they have taken advantage of the different states of motion of the earth in its orbit around the sun, never has such a test been done which is relative to another inertial reference system.

Of course one may say that although these experiments were performed with the earth as the reference system, the earth itself was constantly changing its position in the solar system. Therefore, tests done at different times represent tests relative to different reference systems.

We think this point of view misunderstands the intrinsic meaning of the notion of reference system. The different reference systems should at least include this notion in two senses: different objects of reference and different velocity of the reference objects. The existing experiments cover only tests with respect to one reference object, the earth, travelling at different velocities. If it could be understood that these reference systems have already represented all reference systems of inertia, this implies the postulate: different reference systems refer only to the same reference object moving at different velocities, despite whether this reference object is the earth or some other object. But one must be aware that this implied postulate is just the essential part that should be proved when the relativity principle is to be verified.

Therefore it is necessary to carry out experiments in different systems of inertia, and such experiments are entirely achievable under today's technical conditions. For example, a new Michelson-Morley experiment in Space $\mathrm{Lab}^{\neq 1}$ is one of those feasible experiments.

At present, the velocity that satellites and space craft can reach and the travelling velocity of the earth in the solar system are of the same order; and the development of laser technology has made Michelson's interference experiments relatively easier to realize; besides, with modern measurement techniques we can

\footnotetext{
${ }^{\neq 1}$ Like in Michelson-Morley experiments on the ground, in this experiment, the noninertial effect of Space Lab can also be neglected.
} 
detect a tiny change of optical path in the interferometer. Hence it is quite possible to set a test installation in Space Lab and do Michelson-Morley's experiment again.

By the way, we examine this problem from the point of view of the "aether". If the earth is dragging the aether with it, of course, in a Michelson-Morley experimental installation at rest relative to the earth, no shift of the interference fringes was observed. But in an installation in Space Lab, one can observe a shift. The amount of shifting is:

$\Delta N=(2 L / \lambda)\left(V^{2} / c^{2}\right)$,

where $V$ is the speed of Space Lab relative to the earth, $L$ the length of the light path. Whether such a shift exists can be easily determined with the technology available today.

Suppose such rapidly moving objects had been available at the beginning of this century, people would certainly not have utilized only the earth (moving in the solar system), and ignored the other moving objects. It is regretted that so far no one has begun to do this possible and important experiment.

This experiment will be the first to examine the re- lativity principle in an inertial system other than the earth; at the same time it will also be a check of the principle of constancy of the velocity of light.

Finally, we would like to indicate there is no reason to conclude a priori that the experimental results in another inertial system will certainly confirm the relativity principle, since up to now, no such experiment has been performed. Especially, we consider Sagnac's rotating mirror test [1]. Although Sagnac's experiment is carried out in a rotating system, one point is common to Sagnac's and the suggested experiment, that is, the receiver of light is moving relative to the earth. The real situation could be this: so long as the receiver of the light is moving relative to the earth, the shift of the interference fringes will exist. Thus, the experiment in Space Lab might also find a shift of the interference fringes. This would lead to the failure of the relativity principle in the special theory of relativity.

\section{Reference}

[1] M.G. Sagnac, L'éther lumineux démontré par l'effet du vent d'éther dans un interféromètre en rotation uniforme, C.R. Acad. Sci. (Paris) 157 (1913) 708. 